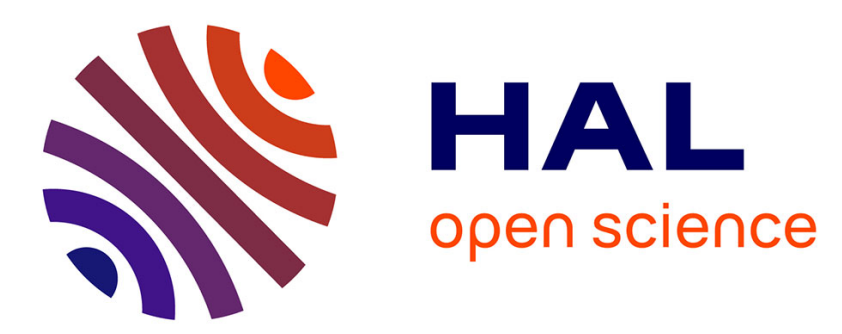

\title{
De la Justice en Amerika: un cas douteux de procès pour harcèlement moral et discrimination chez Kafka
}

Frédérique Leichter-Flack

\section{To cite this version:}

Frédérique Leichter-Flack. De la Justice en Amerika: un cas douteux de procès pour harcèlement moral et discrimination chez Kafka. Les Cahiers de la justice, 2011, 1, pp.173-183. 10.3917/cdlj.1101.0173 . hal-01769188

\section{HAL Id: hal-01769188 \\ https://hal.parisnanterre.fr/hal-01769188}

Submitted on 4 Mar 2019

HAL is a multi-disciplinary open access archive for the deposit and dissemination of scientific research documents, whether they are published or not. The documents may come from teaching and research institutions in France or abroad, or from public or private research centers.
L'archive ouverte pluridisciplinaire HAL, est destinée au dépôt et à la diffusion de documents scientifiques de niveau recherche, publiés ou non, émanant des établissements d'enseignement et de recherche français ou étrangers, des laboratoires publics ou privés. 


\section{De la justice en Amerika : un cas douteux de procès pour harcèlement moral et discrimination chez Kafka}

Article publié dans la revue Les Cahiers de la Justice n²011/1, Dalloz, p. 173-183

Frédérique Leichter-Flack

Amerika ou le Disparu, le premier roman de Kafka (1912), raconte les aventures américaines d'un jeune homme de 16 ans, Karl Rossmann, qui débarque de sa Bohème natale pour refaire sa vie en Amérique, après avoir été expulsé par ses parents. Le premier chapitre, intitulé «Le Soutier » ${ }^{1}$, est pourtant presque entièrement consacré à un étrange procès intenté pour harcèlement moral et discrimination ethnique par un personnage secondaire - le dit soutier - qui disparaît ensuite définitivement, après avoir occupé le devant de la scène pendant les trente premières pages du roman. Faux départ ? Pas exactement, car l'épisode du soutier va précipiter le destin de Karl au «pays des possibilités illimitées », permettant à l'intrigue principale de prendre son envol : au début du chapitre, Karl s'apprête à débarquer, anonyme et sans ressources, dans le port de New York, à la fin du chapitre, il quitte le bateau dans une chaloupe privée, en compagnie de son oncle Jakob, richissime sénateur de New York, tandis que le soutier est abandonné à son triste sort. Entre temps, en retardant son débarquement pour chercher son parapluie oublié dans les profondeurs du navire, Karl a fait la connaissance d'un soutier, l'a écouté se plaindre, dans sa cabine, du traitement discriminatoire qu'il subit de la part de son chef Schubal, l'a accompagné dans la cabine du commandant pour lui servir d'avocat, a assisté à sa confusion et tenté de prendre sa défense, puis, bien malgré lui, s'est vu débouté de sa cause et renvoyé à son propre destin glorieux. Or, c'est l'audience du soutier - ou, plus précisément, son échec - qui déclenche les retrouvailles de Karl avec son oncle d'Amérique : c'est en effet parce le sénateur Jakob s'ennuie pendant le pénible discours du soutier, qu'il sort son petit carnet pour se distraire et se met, par jeu, à comparer les éléments du portrait robot de Karl dont il se trouve disposer, au visage du jeune homme qui s'agite sous ses yeux.

La troublante scène de procès ne serait-elle là, en somme, que pour permettre les retrouvailles du neveu et de l'oncle, et faudrait-il en relativiser l'importance ? Il s'y passe pourtant quelque chose, quelque chose de suffisamment important pour que Kafka, qui n'a presque rien publié de son vivant, ait jugé nécessaire d'assumer la parution de ce premier chapitre sous la forme d'une nouvelle autonome ${ }^{2}$. Ne faudrait-il pas considérer que Karl reçoit ici, à travers l'expérience du procès du soutier, sa première leçon américaine ? Il y en aura d'autres, et de tout aussi rudes, dans la suite d'un roman placé dès sa première page sous le signe d'une Liberté brandissant un glaive au lieu d'un flambeau. De la justice en Amerika : cet épisode douteux de procès pour harcèlement moral et discrimination nous fournira aussi un opérateur de lisibilité possible des enjeux politiques et sociaux de notre démocratie libérale, que la scène «amerikaine » kafkaïenne porte à incandescence. Le recours mesuré, ici, à ces concepts juridiques évidemment anachroniques de «discrimination » et de «harcèlement moral » à propos d'un texte de 1912, se propose aussi d'ouvrir la voie d'une relecture de

\footnotetext{
${ }^{1}$ Franz Kafka, Amerika ou le Disparu, éd. GF, traduction B. Lortholary, p. 17 à 51. Toutes les citations données dans cet article sont empruntées à cette édition.

${ }^{2}$ Ce premier chapitre est en effet paru, du vivant de Kafka, sous la forme d'une nouvelle portant le titre « Le Soutier ». Le reste du roman américain de Kafka, inachevé, et destiné par l'auteur à la destruction comme tous ses autres manuscrits non publiés à la date de sa mort, a été édité à titre posthume, à l'initiative de l'ami et exécuteur testamentaire Max Brod.
} 
Kafka à la lumière et pour l'éclairage des débats d'aujourd'hui, à commencer par celui interrogeant le consensus instable sur les questions de justice sociale au fondement de notre modernité.

Il faut donc relire rapidement la scène, avant d'en interroger la portée. Dans la cabine $\mathrm{du}$ soutier, tout démarre comme un enfantillage. Alors que les passagers du bateau commencent à débarquer dans le port de New York, le naïf Karl Rossmann a confié sa valise à un parfait inconnu, pour partir à la recherche de son parapluie dans les entrailles du bateau. Il rencontre alors, barricadé dans sa cabine, un soutier occupé à ruminer sa rage et son humiliation, qui, submergé par ce flot d'émotions négatives, se plaint à lui du harcèlement discriminatoire que son chef Schubal lui fait subir. La rencontre entre les deux personnages prend d'abord des allures de dialogue de fous, mais Karl, qui se cherche une cause, avec la passion de la jeunesse, encourage son nouvel ami à réclamer justice, et les voilà tous deux qui frappent à la porte de la cabine du commandant. Une scène de théâtre se déploie alors : Karl et le soutier sont comme deux enfants fébriles qui viennent déranger, au moment le plus inopportun, les adultes au travail, avec leurs querelles de justice infantiles. Les voici d'abord remis à leur place, renvoyés par le caissier principal à leur insignifiance avant même d'avoir pu aborder le commandant. Le soutier se résigne à renoncer. Mais c'est compter sans Karl le justicier : le voici qui, faisant barrage de son corps, prend fait et cause pour le soutier, non tant parce qu'il est convaincu que sa cause est juste, mais sans doute parce qu'elle le devient d'avoir été balayée ainsi d'un revers de la main par sa hiérarchie. Personne ne l'a commis, mais Karl se commet lui-même avocat du soutier, et sa première intervention est pour reformuler les plaintes du soutier en une plainte pour préjudice. D'une frustration boudeuse, d'une rage humiliée, Karl fait une affaire que l'on peut porter en justice : « Je me permets de dire, commença-t-il alors, qu'à mon avis l'on a porté tort à M. le soutier. Il y a ici un certain Schubal qui est tout le temps après lui. Il a servi sur de nombreux bateaux, qu'il peut tous vous citer, en donnant toute satisfaction, il est travailleur, il prend sa tâche à cœur et l'on ne voit vraiment pas pourquoi il s'en serait mal acquitté précisément sur ce bateau, où pourtant le service n'est pas excessivement difficile, comme par exemple sur les voiliers de commerce. Il ne peut donc s'agir que de calomnies, si son avancement se trouve bloqué et si l'on lui refuse l'estime à laquelle il devrait normalement avoir droit. Je me suis contenté d'évoquer les grands traits de cette affaire, l'intéressé vous exposera lui-même le détail de ce dont il se plaint. »

Clair, net, percutant, Karl attaque droit au but, en dénonçant une injustice, un tort, un préjudice. Pas un mot cependant sur l'accusation de discrimination ethnique qui constituait l'essentiel des récriminations du soutier contre son chef : en bon avocat, Karl a su faire le tri dans la plainte de son client. Et il obtient une première victoire : tout le monde interrompt son travail, le caissier principal est même obligé de venir se défendre. Celui-ci tente bien d'abord de discréditer le plaignant, invitant le commandant à ne pas prêter attention à cet "homme bien connu pour chicaner sans cesse ». Mais emporté par son exaspération, il commet alors l'erreur de s'adresser directement au soutier ( « je vais vous dire une bonne chose ... »), pour le renvoyer à son infériorité sociale, donnant ainsi l'impression de régler un compte personnel. Grave défaut de stratégie que de contester ainsi, au lieu de la plainte elle-même, le droit du soutier à la porter devant le commandant: le caissier principal donne prise au soupçon d'abus de pouvoir et de complicité avec l'injustice. Karl avait élevé l'affaire au niveau d'une question de justice, et le commandant, du coup, ne peut qu'y être sensible. Même si l'enjeu est tout petit, cela devient une question de principe. Le commandant fait taire le caissier principal, et donne la parole au soutier, acceptant de jouer le rôle du juge arbitre de 
bonne volonté et impartial : «Ecoutons donc cet homme. De toute façon, je trouve de plus en plus que ce Schubal en fait un peu trop à sa tête ; ce qui ne signifie pas pour autant que je me prononce en votre faveur. »

C'est là que les choses, bien engagées pourtant, commencent à se gâter. La suite est racontée à travers les yeux de Karl. Très vite, le soutier s'égare et s'emballe, et la narration en style indirect libre reproduit pour le lecteur l'effet de ses paroles tel que Karl le ressent et devine que les autres le ressentent aussi. C'est une accumulation de reproches sans ordre. $\mathrm{Au}$ lieu de formuler en quoi lui a été spolié de ce à quoi il avait droit, le soutier s'acharne sur Schubal, et transforme la plainte pour préjudice portée en justice par son avocat, en une dénonciation passionnelle, à la limite de la paranoïa. Ce qu'il dit sur Schubal le discrédite, lui, plus encore que Schubal. L'effet sur l'auditoire ne se fait pas attendre: l'irritation est générale, et très vite, on cesse d'écouter le soutier : "bientôt la voix du soutier ne s'imposa plus dans toute la pièce ; on pouvait craindre le pire ». Karl lui-même décroche : son regard s'évade à travers le hublot vers la vie du port. Le soutier, que plus personne n'écoute, continue à s'échauffer, tel un autiste enfermé dans son outrage, qui aurait perdu conscience de son environnement. Karl fait alors, dans l'urgence, une analyse très juste de la situation : « un petit moment de plus, et ils pouvaient fort bien être tous deux flanqués hors du bateau », il faut intervenir de toute urgence. Il n'y a pas lieu, pour Karl, de mettre en doute la légitimité de la colère du soutier, mais il voit clairement combien cette « révolte qui ne connaissait plus de limites » le dessert. Le voici qui tente alors de remettre le soutier sur le bon chemin : « Il vous faut raconter cela plus simplement, plus distinctement, le Commandant ne saurait en tenir compte sous la forme où vous le racontez. » Mais dans le même moment, Karl comprend que c'est impossible: «certes, le soutier s'arrêta net en entendant cette voix connue, mais ses yeux étaient tout embués par les larmes de son honneur blessé, de ses souvenirs terribles, de son extrême détresse présente, et il n'était plus capable de bien distinguer Karl. Comment aurait-on voulu - Karl s'en rendit compte en silence, face à cet homme à présent silencieux , comment aurait-on voulu que tout d'un coup, maintenant, il changeât aussi de discours, alors qu'il avait l'impression d'avoir déjà exposé tout ce qu'il y avait à dire sans éveiller le moindre écho, et en même temps de n'avoir rien dit, sans pouvoir pourtant exiger de ce monsieur d'écouter tout une nouvelle fois encore ? » La tension monte encore d'un degré, car le soutier retourne sa colère et sa frustration contre Karl ; tout le monde est exaspéré, la catastrophe est imminente.

Alors intervient une première péripétie, avec l'arrivée de Schubal. L'air dégagé et fringant, un registre à la main, Schubal est en contraste flagrant avec le soutier : solide, doué d'une parfaite maîtrise de soi, et d'un «langage net et viril », il parle clair et court performance ostensible qui ne peut qu'écraser le soutier décomposé, et qui nous le rend d'emblée détestable. Mais si la force qui se dégage de Schubal ne nous est pas sympathique, de fait, elle soulage tout le monde, et jusqu'au lecteur, par contraste avec la logorrhée pathétique et pesante d'un fou qui n'a plus aucune tenue («à voir changer les expressions de l'assistance, on aurait pu croire que c'était la première fois depuis longtemps qu'on entendait des sons humains »). Ce discours en apparence solide trahit cependant ses failles, que l'avocat Karl relève immédiatement, refaisant le procès dans sa tête (" pourquoi le premier mot précis qui lui était venu à l'esprit était-il celui de «malhonnêtetés »? Est-ce que, peut-être, l'accusation n'aurait pas dû partir de là, plutôt que de ses préjugés nationaux ?»), mais comment le soutier en ferait-il désormais usage ? Karl s'apprête donc à intervenir pour une ultime tentative de suppléer à son client déficient.

C'est alors que la fiction devient vraiment cruelle. Pour la deuxième fois depuis le début de la scène, le dénommé $\mathrm{M}$. Jakob, en conversation avec le commandant, demande à Karl son nom. Le coup de théâtre des retrouvailles de l'oncle et du neveu crée alors, dans 
cette atmosphère lourde, un soulagement de comic relief, aux frais de l'adolescent Karl. La scène de procès politico-sociale laisse alors place à un épisode de vaudeville ou de mélodrame. Voici que l'oncle se met à raconter à l'assistance amusée toute l'histoire de l'affaire de Karl avec la bonne, à l'origine de l'expulsion parentale. L'intrigue, en réalité, a retrouvé son fil, et le soutier lui est devenu inutile : lui-même comprend immédiatement qu'il est déjà sorti de l'histoire, et en prend acte en félicitant Karl de sa bonne fortune. Ce dernier a un peu plus de mal à tirer les conséquences qui s'imposent de ce changement de situation. Le sénateur doit insister pour persuader son neveu de cesser de "s'immiscer de surcroît sans nécessité aucune dans la querelle subalterne entre deux machinistes et d'en faire un événement », et c'est en larmes, agrippé au soutier, que Karl fait ses adieux à l'homme dont il avait embrassé la cause, et qu'il doit finalement abandonner à son sort.

«Ne te méprends point sur la situation, c'est peut-être une question de justice, mais c'est en même temps une question de discipline », explique le sénateur à son neveu ; « conçois la situation qui est désormais la tienne », lui enjoint-il encore ; "comprenez donc, jeune homme, la chance que vous avez [...] essayez de comprendre cela, autant qu'il est possible sur le moment, et ressaisissez-vous ", lui avait déjà répété le commandant. L'insistance de ces deux adultes à enjoindre Karl de grandir, de cesser de faire l'enfant, signifierait-elle quelque chose de plus qu'une simple invitation à profiter de l'aubaine de ce nouveau statut social qui s'offre à lui ? Cesser de faire l'enfant, serait-ce aussi cesser de ne voir que l'injustice là où il y aurait aussi autre chose à apercevoir, autre chose que Karl doit apprendre d'urgence à reconnaître, s'il veut espérer faire son chemin en Amerika ? Quel est donc ce message que le sénateur Jakob veut faire comprendre à ce neveu trop sensible à la brûlure de l'injustice et prêt à tout interrompre pour y remédier, à ce jeune homme abandonné qui s'est accroché à la justice comme à une bouée de sauvetage au moment où il se sentait seul et sans ami ? Quelle puissante leçon la scène de procès du soutier a-t-elle donc administrée à Karl, dans le cadre de ce roman de formation paradoxal qu'est Amerika, pour que par la suite, lui qui était si prompt à s'enflammer contre l'injustice faite à autrui, non seulement ne tentera jamais rien pour réclamer réparation des nombreux préjudices et injustices dont il sera lui-même victime, mais encore ne se permettra jamais le moindre sentiment de révolte?

Il est temps de revenir un peu sur l'affaire portée devant le juge commandant. Que penser du soutier? Le personnage n'est pas sympathique. Attire-t-il même la pitié du lecteur? Rien n'est moins sûr. Si l'on devait choisir, entre lui et Schubal, qui choisirait-on ? Plus la scène avance, et plus on est du côté de Schubal, tout en trouvant Schubal absolument détestable. C'est que le débat s'est déplacé : très vite, il ne s'agit plus de décider qui a tort, et qui a raison, ou de savoir si le soutier a été traité injustement, si un tort lui a été fait, s'il a été en effet discriminé, mais seulement de se demander, finalement, ce qu'on peut bien faire d'un individu pareil...Et c'est là peut-être tout le risque de la justice : quand on porte plainte, on attire l'attention sur soi, le juge ne fera pas qu'examiner la plainte, il scrutera la personnalité du plaignant, les mérites comparés, les circonstances et l'environnement. Une fois lancée, la justice s'autorise à tout juger, à fournir un verdict global, à valider les choix du destin, ou à les défaire - c'est dans sa main.

Pourquoi, alors, le soutier échoue-t-il à ce point? De quoi est tissé son échec ? Plusieurs dimensions entrent en ligne de compte, qu'il importe de démêler. D'abord, la scène est évidemment traversée de part en part par la problématique sociale. Il est difficile pour le soutier d'être là, dans la cabine du commandant, "pauvre homme en butte aux grands ». Comment saurait-il articuler ses griefs, lui qui - cela saute aux yeux - ne possède pas les 
codes pour s'exprimer devant un tel public, lui qui est si manifestement dépourvu du capital culturel nécessaire pour saisir la chance que le commandant lui offre de court-circuiter sa hiérarchie? Pire encore, comment se libérerait-il de ce carcan social qui le dessert, quand ce dont il a à se plaindre insiste sur le fossé social au lieu de le combler ? Sa querelle à lui est au ras du sol, et il est bien obligé, pour l'exprimer, de citer les noms de tous les machinistes subalternes, même si, comme Karl croit utile de le lui faire remarquer, le commandant n'en a que faire et ne saurait connaître «tous les hommes des machines et tous les petits chasseurs par leur nom, voire leur prénom ». Ensuite, le soutier fragilise très certainement son argumentaire en accusant Schubal le Roumain de discrimination ethnique contre les Allemands de l'équipage. L'argument du racisme est à double tranchant : il faut être sûr de soi pour le manier, car il ne peut que susciter, presque automatiquement, un réflexe de soupçon. Son seul tort serait d'être allemand? Voire... L'accusation de discrimination ethnique oblige le juge, pour la vérifier, à se demander si on n'aurait pas d'autres bonnes raisons de «discriminer » cet individu, autre que sa nationalité, à examiner tout ce qui, dans le plaignant, pourrait attirer plus «légitimement» l'inimité. Il faudrait être parfait et irréprochable par ailleurs ; mais qui l'est?

Enfin, reste le fond du problème : le problème de souffrance au travail, la plainte pour harcèlement moral, dirait-on aujourd'hui. Le soutier souffre avant tout de ne pas être reconnu dans son travail, de ne pas être respecté. Le voilà qui donne tout de suite l'exemple flagrant qui le trahit: «M. Schubal expulsait le soutier de la salle des machines et lui faisait nettoyer les cabinets, ce qui n'est tout de même pas son travail !» Il est humilié, et le propre de l'humilié, c'est qu'il vit toute nouvelle demande, toute négligence de son humiliation comme une humiliation supplémentaire... Et c'est là qu'est l'échec programmé du soutier. Car comment prouve-t-on qu'on a été victime de harcèlement moral, sinon en faisant la preuve de sa propre démolition psychique, et en listant les innombrables micro-humiliations subies ? Or, ces humiliations sont, par définition, toutes petites : les redire, c'est s'humilier à nouveau, se donner à voir sous son plus mauvais jour. Comment, alors, faire la preuve par son effondrement psychique, tout en donnant tout de même envie au juge d'assumer la responsabilité de rétablir la dignité atteinte en réparant le tort ? Mais si celui-ci se dit, en son for intérieur, que la personne est tellement défaite qu'elle n'est plus bonne à rien ?

Qu'est-ce donc que la dignité ? Ne faut-il pas tout de même un reste de fierté pour faire reconnaître sa dignité ? A partir de quand l'effondrement d'une personne l'en prive-telle complètement ? C'est, on s'en souvient, tout le problème du poème liminaire que Primo Levi a placé à l'ouverture de Si c'est un homme. Car il est facile d'écraser sans limites des hommes à qui l'on a préalablement ôté toute dignité. C'est l'aporie de la dignité, une qualité intrinsèque à tout homme, en-dessous de toutes les autres qualités, une sorte de plus petit commun dénominateur à l'humanité, mais tandis que c'est justement quand toutes les autres qualités d'une personne ont disparu que la reconnaissance de sa dignité lui devient le plus nécessaire, la dignité disparaît aussi... Si la dignité humaine est ce qui reste de droit à tout homme quand il a perdu tout le reste, il n'est pourtant pas facile de la lui reconnaître quand, précisément, il a perdu tout le reste. La dignité est-elle donc un droit de l'homme ou ce qui donne à l'homme la jouissance des droits de l'homme ? C'était déjà le problème que formulait Hannah Arendt, à propos des droits de l'homme à reconnaître aux apatrides de l'entre-deuxguerres, dans un chapitre célèbre des Origines du Totalitarisme ${ }^{3}$.

\footnotetext{
${ }^{3}$ «Si un être humain perd son statut politique, il devrait, en fonction des conséquences inhérentes aux droits propres et inaliénables de l'homme, tomber dans la situation précise que les déclarations de ces droits généraux ont prévue. En réalité, c'est le contraire qui se produit. Il semble qu'un homme qui n'est rien d'autre qu'un homme a précisément perdu les qualités qui permettent aux autres de le traiter
} 
«Que va-t-on faire du soutier ? », insiste Karl. Mais que peut-on faire d'un soutier pareil ? Et comment le bateau fonctionnerait-il avec des soutiers comme celui-là ? Telle est sans doute la question que le commandant, seul juge à bord, doit se poser depuis le début de l'audience. Peut-on réparer l'injustice probablement subie par le soutier en le réhabilitant sur ce bateau, lui que les humiliations accumulées ont rendu dysfonctionnel, fou de détresse, répugnant de malheur? Il faut prendre au sérieux la réponse du sénateur à la question de Karl : «on va en faire ce qu'il mérite ». Tout se passe comme si, en lui faisant subir ce qu'on lui fait subir, on prouve qu'il méritait qu'on le lui fasse subir. Comme si le soutier ne méritait pas autre chose que ce qui lui arrive, comme si son effondrement justifiait, a posteriori, la discrimination que lui a fait subir Schubal, la mise à l'écart, la disqualification professionnelle, sociale, et morale.

La logique du harcèlement moral ressemble à celle du bizutage, soit une épreuve cruelle, mais qui vise à tester une capacité de résistance psychique, une solidité qualifiante pour la fonction à exercer. S'effondrer, c'est se disqualifier. A Karl qui déclarait qu'on a privé le soutier de ce à quoi il avait droit, l'examen de son cas par la fiction répond qu'il n'a eu que ce qu'il méritait, et donne presque raison, rétrospectivement, au détestable Schubal. Car tel que la justice l'a examiné, tel qu'il se découvre sous nos yeux, comment le soutier mériteraitil un autre traitement que celui dont il se plaint ? L'incapacité du soutier à se défendre, de par sa souffrance même, fait perdre toute légitimité à sa cause, et le fait apparaître comme irrécupérable, définitivement incapable. Sa détresse le rend fou, et cette folie lui ôte ses droits. N'y a-t-il pas, d'ailleurs, quelque chose de ce découragement, de cette répulsion troublante et inavouable, dans la réaction à fleur de peau de Karl face au soutier apathique et comme résigné à sa propre défaite : « - Mais pourquoi ne dis-tu rien ? lui dit-il. Pourquoi te laisses-tu faire sans réagir ? [...] Mais il faut que tu te défendes, que tu dises oui ou non, sinon les gens ne soupçonneront pas la vérité. »

A la question de Karl, « que va-t-on faire du soutier, maintenant ? », le sénateur Jakob ne fait alors que répondre tout haut, et sans complexe, ce que tout le monde pense tout bas : «On va en faire ce qu'il mérite, et ce que le Commandant jugera bon qu'on en fasse. Je crois que nous en avons assez de ce soutier, plus qu'assez ». Et de répliquer, à l'objection de Karl («Mais là n'est pas le problème, c'est une question de justice ») : «c'est peut-être une question de justice, mais c'est en même temps une question de discipline ». La justice n'est pas seule en piste, il faut encore que la discipline, que les règles du jeu, soient respectées, c'est-à-dire que le système social puisse fonctionner. Ce n'est pas une défense de l'ordre social, car la société «amérikaine »s'accommode d'une très grande fluidité, comme cet ascenseur qui ne cesse de monter et de descendre dont Karl aura la charge, quelques chapitres plus loin, quand il sera embauché comme liftier à l'hôtel occidental. Nul besoin que chacun reste à sa place; mais il faut que chacun tienne sa place, joue son rôle, assure la fonction dont il est chargé au moment présent. Et c'est là la principale critique formulée contre le soutier, une critique disqualifiante contre son ethos : il est « chicaneur », au lieu de travailler, il passe son temps à récriminer, à protester, à contester, à examiner si ce qu'on lui demande de faire fait bien partie de son contrat de travail, si on n'est pas en train de tenter d'abuser de lui... Jamais il n'acceptera de nettoyer les toilettes, alors qu'il faut bien, de temps en temps, en passer par là, pour gagner le droit d'en être définitivement dispensé... Qui voudra embaucher un chicaneur? Celui qui se plaint ne se disqualifie-t-il pas pour l'avenir? Le problème est aujourd'hui bien connu : comment l'exploitation de stagiaires ferait-elle l'objet d'un traitement judiciaire?

comme leur semblable» Hannah Arendt, Les Origines du Totalitarisme, Paris, Gallimard, Quarto, 2002 , p. 604. 
On peut pourtant obtenir justice en Amerika, et même recevoir des compensations au centuple. Encore faut-il faire la preuve de sa valeur. Tout se joue, pour le soutier, en quelques secondes. Son audace aurait pu être payante. Mais il échoue, et fait ainsi la preuve qu'on devait en effet se débarrasser de lui. Etrange et effroyable inversion de l'ordre de la rétribution. Son incapacité à supporter l'injustice sans s'effondrer le rend, en quelque sorte, indigne d'être respecté. Subir l'injustice implique pourtant, chez tout le monde, un risque d'effondrement psychique. Plus l'injustice et son humiliation sont petites, plus le risque est grand. Quand l'injustice est immense et écrasante, on peut toujours vivre de l'énergie de sa révolte, de son désir de vengeance. Mais les petites histoires de harcèlement moral ne façonnent pas des comtes de Monte Christo. Celui qui s'y laisse prendre y perd ses moyens, tout simplement, et jusqu'à la capacité de prouver aux autres qu'il mérite de faire valoir ses droits au respect. Cercle vicieux de l'écrasement : ce n'est pas parce qu'il souffre qu'on doit lui donner raison.

Y aurait-il donc des hommes qui ne mériteraient pas le respect ? qui ne mériteraient rien d'autre, pendant qu'ils se répandent en plaintes, en larmes et en sueur d'outrage, que l'irritation, l'ennui et l'exaspération, que cette réaction impitoyable du sénateur qui sort son petit carnet pour se distraire en attendant que l'homme veuille bien se taire ? Chez Kafka, la scène américaine est d'une très grande dureté. Il faut être solide pour y résister. Sinon, on s'écroule, on y est englouti, on y « disparaît ». D'ailleurs, les «disparus », en Amerika, n'ont même pas de tombe, mais rien d'autre qu'un trou dans l'eau qui se referme, un évanouissement dans la foule anonyme des miséreux errants. Quelques chapitres plus loin, le roman évoque une scène de suicide. C'est Thérèse, secrétaire à l'hôtel occidental, qui raconte la mort de sa mère, des années auparavant, quand, petite fille, elle se souvient d'avoir erré toute une nuit de tempête, avec sa mère, dans le froid et la pluie, chassées de partout, sans sommeil ni abri. Au matin, sa mère épuisée l'avait trainée jusqu'à un chantier où elle s'était faite embaucher, était montée tout en haut d'un échafaudage, et avait sauté. Et la fille, qui a grandi, et travaille désormais très dur, jour et nuit, dans l'univers impitoyable de l'hôtel occidental, raconte cela sans se plaindre, sans la moindre trace de révolte ou de colère, sans le moindre laisser aller émotionnel.

Bien sûr, la démocratie libérale à la Kafka, ce n'est pas que cela : au «pays des possibilités illimitées », l'oncle Jakob raconte aussi comment il a fait fortune, à partir de rien, à force d'autodiscipline. Assurément, la tête de Schubal était à portée de main du soutier, pourvu qu'il se fût montré capable de s'en saisir. Tout se passe en fait comme si, en Amerika, seuls ceux qui ont supporté sans s'effondrer l'épreuve de l'injustice, peuvent réussir : on n'y a pas de chance, on n'y a que du mérite. La valorisation des capacités d'endurance psychique à la limite du supportable pour un lecteur occidental moderne - assoit la méritocratie « amérikaine » sur la résistance à l'injustice, à la dureté du sort. Grandir en Amerika, grandir en américain, chez Kafka, c'est comprendre qu'il n'y a nulle justice à attendre de la vie et qu'on ne sera pas armé pour la vivre si la moindre injustice nous arrête. La réflexion sur le mérite remplace et annule celle sur la réparation du préjudice. Mais en Amerika, la justice règne cependant : c'est elle qui accueille, glaive en main, les immigrés qui viennent tenter leur chance sur ses rives, toujours prête à trancher, après avoir scruté les mérites respectifs des plaignants, fouillé dans les chairs à nu des parties en présence, sans appel possible.

D'autres signes pourtant plaident, en parallèle, pour une autre lecture de cet épisode : cela ressemble fort, après tout, à un bizutage dans lequel un étranger un peu trop sensible se mêlerait d'intervenir. Car personne, à part le soutier bien sûr, et le tout jeune Karl venu d'Europe, ne semble prendre cela au tragique ; l'arrivée des témoins se fait dans un joyeux bazar ; une fille des cuisines affuble même le matelot dépêché auprès du commandant de son 
tablier. «Ce n'était pas bien méchant, c'est pour s'amuser !», semblent dire les visages rieurs des témoins de Schubal, faisant des signes d'adieu à Karl et à son oncle à travers les fenêtres de la cabine du commandant. Tout le monde semble d'accord pour considérer ce recours à la procédure judiciaire comme cocasse et saugrenu - sauf le commandant au début du chapitre, parce qu'il se demande si ces abus n'ont pas été trop loin, s'il n'est pas temps d'y mettre un frein. Mais l'examen de la personnalité du plaignant va, en somme, le convertir aux vertus du bizutage, et le convaincre de lasser l'affaire se régler entre les mains de ses subalternes (dont le soutier devine qu'ils seront chargés de le mettre dehors). Incongruité d'un procès pour bizutage ! Celui qui proteste, qui se mêle de dénoncer, non seulement prouve qu'il n'a rien compris au jeu, mais se met définitivement hors jeu lui-même. Tout pourrait bien s'être passé ici, comme dans cet autre roman de Kafka, le Château, quand, après avoir usé ses nerfs et épuisé toutes ses ressources mentales pour lutter contre son insaisissable adversaire et accéder au Château, K. apprend de ses assistants, les grotesques Arthur et Jérémie, qu'ils lui avaient été envoyés par le Château lui-même non pour l'assister, mais pour le distraire, le dérider, lui servir de clowns, lui qui « prend tout tellement au sérieux alors que ce n'est pas si grave »...!

Difficile, alors, de décider laquelle de ces deux lectures est la plus cruelle...Toutes deux nourrissent sans doute un commun malaise de lecture, qui emporte peut-être toute la portée éthique et politique de ce non-événement en obligeant le lecteur à affronter un problème inaperçu. La littérature ne donne pas à la politique le moyen de le résoudre, pas plus qu'elle ne s'en donne le moyen : le trou dans l'eau que fait le malheureux soutier en tombant à la mer se referme vite, et le personnage disparaît de la fiction comme s'il n'avait jamais existé. "Tout se passait vraiment comme s'il n'y avait plus eu de soutier », conclut Karl à la fin du chapitre. Mais l'espace d'un instant, en conscience, le laissé-pour-compte aura apparu, silhouette have et fantomatique, pantin gesticulant tout en sueur d'efforts pour se faire admettre, assez cependant pour persister comme une trace indélébile dans le sillage de cette humanité qui ne peut plus rien pour lui. 